\title{
Prosthetic Rehabilitation of Nasal Septal Defect with Magnet Retained Nasal Septal Prosthesis
}

\author{
Hina Zafar Raja ${ }^{1}$, Maryam Mumtaz ${ }^{1}$ and Muhammad Nasir Saleem ${ }^{2}$ \\ ${ }^{1}$ Department of Prosthodontics, Institute of Dentistry, CMH Lahore Medical College, Lahore, Pakistan \\ ${ }^{2}$ Department of Operative Dentistry, Institute of Dentistry, CMH Lahore Medical College, Lahore, Pakistan
}

\begin{abstract}
Nasal septal defects may present with no clinical symptoms to dryness, nasal obstruction, pain, mucosal injury, crusting, epistaxis, rhinorrhea, nasal twang in speech, hyposmia, and breathing difficulties. Prosthetic rehabilitation of these defects may lead to improvement in these conditions. This case report describes the construction of a custom-made two-piece, magnet-retained nasal prosthesis fabricated in heat-cured acrylic resin. Intra-nasal and extra-nasal alginate impressions were splinted with soft plaster. Sequential model pouring was done for convenient access to the complicated intra-nasal anatomy. Two individual nasal stent waxups, containing two magnets with opposing poles facing each other, were well adapted to their respective medial nasal walls and anterior retentive rounded tips. Waxups were processed in heat-cured acrylic resin and delivered after finishing and polishing. Patient's symptoms were relieved, while maintaining the nasal patency. Such inexpensive method with a tissue-friendly material may prove beneficial for rehabilitation of larger nasal septal defects.
\end{abstract}

Key Words: Nasal septal defects, Rehabilitation, Acrylic resin, Prosthesis.

How to cite this article: Raja HZ, Mumtaz M, Saleem MN. Prosthetic Rehabilitation of Nasal Septal Defect with Magnet Retained Nasal Septal Prosthesis. J Coll Physicians Surg Pak 2021; 31(12):1506-1508.

\section{INTRODUCTION}

Nasal septal perforations may result from iatrogenic, traumatic, neoplastic, caustic, inflammatory, and infectious diseases. ${ }^{1-3}$ These may present with no symptoms to cold intolerance, irritation, secondary rhinorrhea, crusting, epistaxis, mild low grade chondritis, midfacial osteomyelitis along with whistling or foul odour. ${ }^{4,5}$ Conservative management includes saline douching or emollients. ${ }^{5}$ Large defects may require single-piece or two-piece custom-made obturator in acrylic resin, silastic or silicone made conventionally or with digital work flow. ${ }^{6}$ This case report describes prosthetic management of a patient with a nasal septal defect (NSD) due to surgical resection of squamous cell carcinoma (SCC) with a custom-made two-piece, magnet-retained prosthesisfabricated in heat-curing acrylic resin.

\section{CASE REPORT}

A 33 years old female presented with NSD of about $4 \mathrm{~cm}$, due to surgical resection of SCC, 15 years ago (Figure 1a). Surgical closure of defect was never attempted due to large size during this timeperiod.

Correspondence to: Prof. Hina Zafar Raja, Department of Prosthodontics, Institute of Dentistry, CMH Lahore

Medical College, Abdul Rehman Road, Lahore Cantt,

Lahore, Pakistan

E-mail: hinazafarraja@gmail.com

Received: September 25, 2020; Revised: February 09, 2021;

Accepted: May 24, 2021

DOI: https://doi.org/10.29271/jcpsp.2021.12.1506
She experienced pain and difficulty during breathing for the past two years that exacerbated during cold weather. After physical and radiographic evaluation, advice for prosthetic closure via a two-piece, custom-made, magnet-retained acrylic nasal septal obturator was presented to the patient.

Primary impression of nasal cavity was made in medium-body alginate, after applying topical anesthesia and vaseline on mucosa. Gauze pieces were packed within nasal cavity to prevent deeper flow of the material. Mixed alginate impression material was syringed into the nasal cavity via a 10 cc disposable syringe. Match sticks were inserted inside each nares to facilitate removal of impression (Figure 1b).

A self-cure acrylic tray, supported by a stainless wire, was fabricated over primary casts. Custom tray had a U-shaped anterior portion with two prongs for each of the nasal cavity and a handle posteriorly to facilitate removal of impression (Figure 1c). For secondary impression, the custom tray was dipped in molten wax, cooled and then molded within nasal cavity, to support secondary impression material. Secondary impression was taken with medium body addition silicone. Extra-nasal impression was recorded in alginate impression material. It was spread with the help of a spatula. Both intra-nasal and extra-nasal impressions were removed collectively with a splint of soft plaster (Figure 1d). Cast was poured in three steps to allow easier separation. Hard plaster was painted with a brush on the impression surface of extra-nasal impression. 


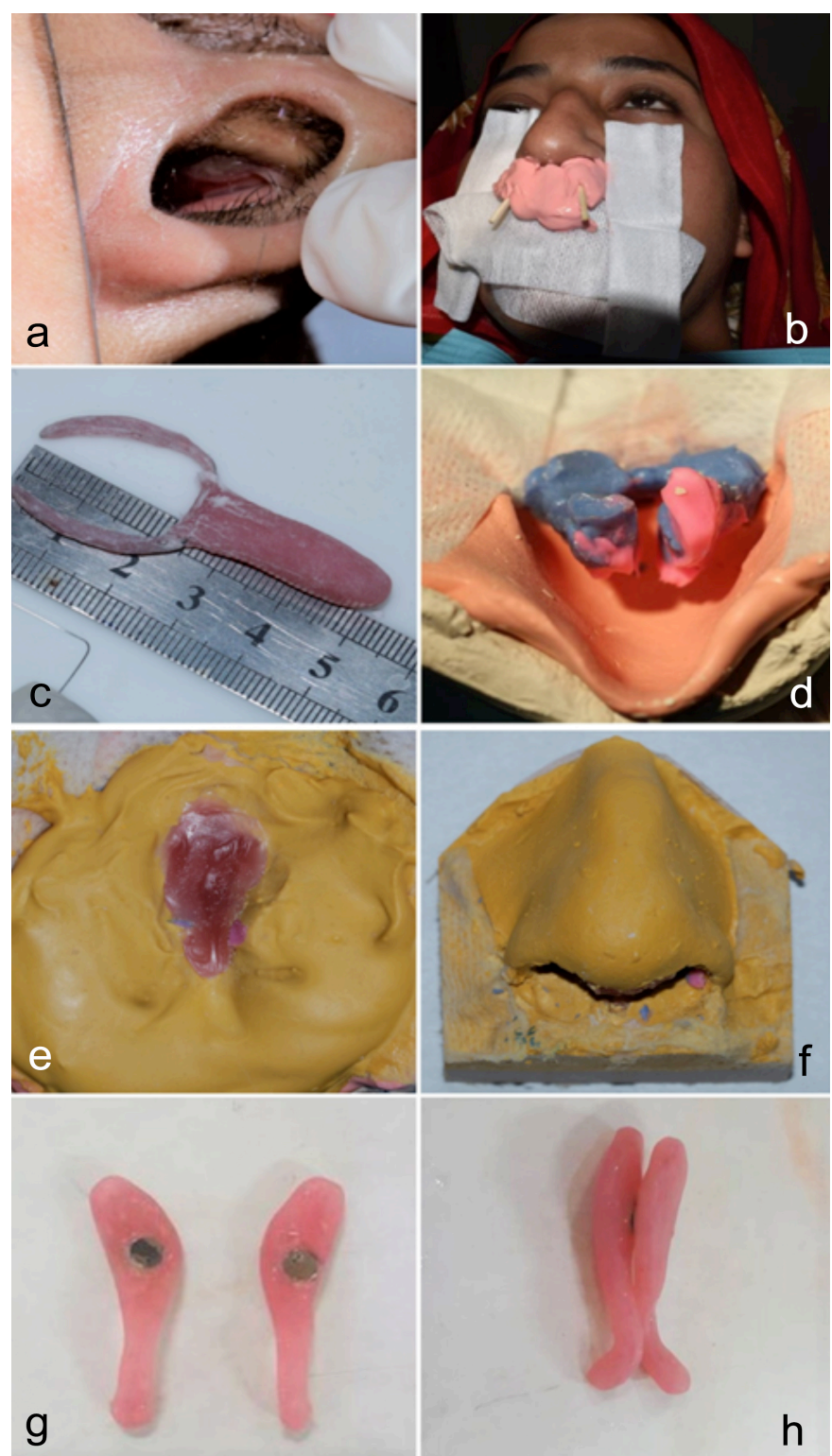

Figure 1: (a) Nasal septal defect; (b) Primary impression in alginate; (c) Self-cure acrylic special tray; (d) Splinted intra-nasal and extra-nasal impressions; (e) Partially poured casts with wax patterns; (f) Cast with separable internal and external nasal parts; ( $g$ ) Finished and polished nasal septal prosthesis; (h) Wax patterns with magnet with opposing poles facing each other.

Then, it was poured in, between the external and internal nasal forms, to support the inner structure of the nasal cavity up to the base of alae of nose. Material wasallowed to setfor smooth application of separating media. Molten wax was poured in to the septal defect cavity of the plaster cast to form an anatomical wax pattern. Hard plaster was then poured to fill up the remaining portion of intra-nasal impression (Figure 1e). The cast was then boxed with the help of cardboard to form its base. The external nasal form was separable from the internal nasal form (Figure 1f). The wax pattern was trimmed and finished as two separate pieces, both adapting medially with each other. The anterior portion of both wax patterns were made ballshaped to adapt them under the tip of nares, to act as a means of mechanical retention. Two magnets with opposing poles facing each other, were inserted in the postero-medial portion of the wax pattern of each side (Figure 1g). After wax trial, both wax patterns were processed individually in metallic denture flask, in heat-cured acrylic resin (Heat cure acrylic resin company: Imperial Dental Supply Co.).

Prosthesis was inserted afterfinishing and polishing (Figure $1 \mathrm{~h}$ ). The patient was counselled about insertion and removal, especially at night-time, to prevent its posterior displacement. The patient reported relief of her symptoms of pain and difficulty in breathing. Follow-up appointments were scheduled to alleviate any possible complaints.

\section{DISCUSSION}

NSDs arise due to bilateral adjoining disruption of the mucoperichondrium and underlying cartilage and/or bone. ${ }^{1}$ Larger defects may be managed prosthetically. ${ }^{7}$ Surgical repair may lead to incomplete closure, impaired healing with creation of an even bigger defect or external nasal deformity with persistence of symptoms. Moreover, the local conditions inside the nasal cavity, age and systemic health of the patient may also preclude surgical management.

Nasal stents are standardised or customised, one-piece or two-piece. They can be fabricated from medical grade silicones, acrylic resin, rubber or plastics. ${ }^{8} \mathrm{Heat}$-cure acrylic resin can be polished to a smooth surface with good durability and lesser tendency for water sorption or mucosal crusting. ${ }^{8}$ Many techniques are proposed for fabrication of customised nasal stents or septal prosthesis, including 3-D printing, to overcome the problem of impression making of the defect area. ${ }^{2}$ However, digital scans of intra-nasal areas might not be readily available globally. In addition, they may require CT scan of the complete head and neck region. ${ }^{9}$

This case report describes an innovative impression technique, including two steps, to achieve a more reliable and precise impression for construction of an accurately fitting prosthesis. It utilises conventional materials and methods. In addition, a three-staged cast pouring for fabrication of nasal prosthesis model was done. Model was poured in sections to permit the fabrication of wax pattern in accordance with anatomical contours of the nasal cavity and septal defect. The pouring of melted wax directly into the defect portion of cast, allowed precise adaptation of pattern as per the dimensions of the defect. It also facilitated easier approach to intra-nasal areas.

The means of retention for nasal septal prosthesis can be adhesives, mechanical retention or magnets. ${ }^{10}$ Two magnets with opposite poles facing each other were able to attract the two portions of nasal septal prosthesis with an effective magnetic force. Anteriorly, mechanical retention was gained with a small bulb placed under the soft tip of nose. This proved better than other adhesives, which can be affected through nasal secretion. Other possible options are wire retention or mandrills for cast section retention. ${ }^{11}$ 
This technique of obturation of nasal septal perforation is effective, economic, utilises local anesthesia, and causes resolution of symptoms whilst retaining the patency of the nasal cavity at the same time, with known bio-compatible materials. ${ }^{12}$ It was effective in relieving the patient's symptoms of pain and breathing difficulty. Thus, fabrication of a custom-made nasal stent directly from an accurate impression is one of the most feasible treatment options for closure of a large nasal septal perforation.

\section{PATIENT'S CONSENT:}

Informed consent was obtained from the patient regarding the publication of this case report.

\section{CONFLICT OF INTEREST:}

The authors declared no conflict of interest.

\section{AUTHORS' CONTRIBUTION:}

HZR: Patient treatment planning, conceptualisation of the 3step cast pouring technique, performance ofall clinical and laboratory steps, follow-up of patient, writing the manuscript.

MM: Assistant in performance of clinical and laboratory steps, picture taking and record keeping, contributor in manuscript writing.

MNS: Evaluation and measurement of the nasal septal defect, patient treatment planning, major contributor in manuscript writing.

All authors read and approved the final manuscript.

\section{REFERENCES}

1. Metzinger SE. Diagnosing and treating nasal septal perforations. Aesthet Surg J 2005; 25(5):524-9. doi: 10. 1016/j.asj.2005.06.002.

2. Passali D, Spinosi MC, Salerni L, Cassano M, Rodriguez H, Passali FM, et al. Surgical treatment of nasal septal perforations: SIR (Italian Society of Rhinology) experts opinion. Acta Otorrinolaringol Esp 2017; 68(4):191-6. doi: 10.1016/j.otorri.2016.10.001.

3. Ohlsén L. Closure of nasal septal perforation with a cutaneous flap and a perichondrocutaneous graft. Ann Plast Surg 1988; 21(3):276-88. doi: 10.1097/00000637-198809 000-00017.

4. Hanci D, Altun H. Repair of nasal septal perforation using middle turbinate flap (monopedicled superiory based bone included conchal flap): A new unilateral middle turbinate mucosal flap technique. Eur Arch Otorhinolaryngol 2015; 272(7):1707-12. doi: 10.1007/s00405-014-3266-1.

5. Blind A, Hulterström A, Berggren D. Treatment of nasal septal perforations with a custom-made prosthesis. Eur Arch Oto-Rhino-Laryngol 2009; 266(1):65-9.doi: 10.1007/ s00405-008-0690-0.

6. Hasan Farook T, Jamayet N. A review of prostheses fabricated for rehabilitation of nasal septal defect using digital workflow. Otorinolaringologia 2020; 70. doi:10.237 36/S0392-6621.20.02254-7.

7. Goh AY, Hussain SS. Different surgical treatments for nasal septal perforation and their outcomes. J Laryngol Otol 2007; 121(5):419-26. doi: 10.1017/S002221510700566X.

8. Goyal MK, Goyal S. Prosthetic rehabilitation of large nasal septal defect with an intranasal stent: A clinical report. Indian J Dent Res 2011; 22(5):719-22. doi: 10.4103/ 0970-9290.93464.

9. Somohano Marquez T. Custom nasal septum prosthesis fabricated from a 3D-printed working model: A clinical report. J Prosthodontics 2019; 28(5):493-6. doi: 10.1111/ jopr.13064.

10. Sashi Purna CR, Annapurna PD, Ahmed SB, Vurla S, Nalla S, Abhishek SM. Two-piece nasal septum prosthesis for a large nasal septum perforation: A clinical report. J Prosthodont 2013; 22(2):143-7. doi: 10.1111/j.1532-849X

11. Nagaraj E, Shetty M, Krishna PD. Definitive magnetic nasal prosthesis for partial nasal defect. Indian J Dental Res 2011; 22(4):597. doi: 10.4103/0970-9290.90309.

12. Sashi Purna CR, Annapurna PD, Ahmed SB, Vurla S, Nalla S, Abhishek SM. Two-piece nasal septum prosthesis for a large nasal septum perforation: A clinical report. J Prosthodont 2013; 22(2):143-7. doi: 10.1111/j.1532849X.2012.00911.x. 